\title{
Effects of a 3D Body Imaging Trigger on Self-Perceived Attractiveness, Self-conscious Emotions and Coping
}

\author{
RACHEL NANEZ, ALYSSA ABREU, MARK D. FARIES, ERIC J. JONES
}

Lifestyle Medicine \& Behavior Laboratory; Department of Kinesiology \& Health Science; Stephen F. Austin State University, Nacogdoches, TX

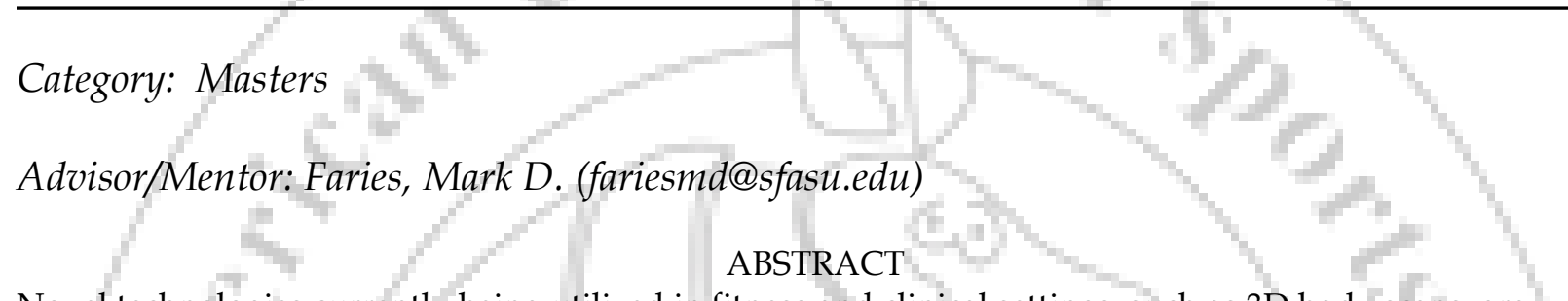

Novel technologies currently being utilized in fitness and clinical settings, such as 3D body scans, are proposed to act as a trigger or spark for weight control behavior. However, other weight-related triggers in women have been shown to produce variation in emotional and weight control responses, and there is limited research on their impact. The purpose of the study was to examine the acute effect of 3D body imaging system (Fit3D) on self-conscious emotions (guilt, shame, pride) and body weight-related coping in a sample of college-aged women $(N=30)$ classified as normal weight $(\mathrm{NW}, n=12)$ or overweight/obese (OWOB, $n=18$ ). To this end, the Body and Appearance-related Self-conscious Emotions Scale (BASES; range: 1-5) and the WEIGHTCOPE (range: 1-7) were used to assess self-conscious emotions and intention to utilize 10 different weight-related coping strategies following a single 3D body scanning session. Body fat percentage $(\mathrm{BF} \%)$ was assessed via Dual-energy $x$-ray absorptiometry (DXA). Following the 3D scan, participants experienced low to moderate feelings of guilt $(3.18 \pm .99)$, shame $(2.67 \pm 1.05)$, authentic pride (2.68 \pm .96$)$, and hubristic pride $(2.49 \pm .87)$. Significant differences $(p<.05)$ were found between participants classified as NW or OWOB, respectively, in shame ( $2.11 \pm .76$ vs. $3.05 \pm 1.07)$, guilt $(2.82 \pm .85$ vs. $3.43 \pm 1.02)$, and authentic pride ( $3.10 \pm .78$ vs. $2.39 \pm .98)$. Increasing physical activity and selfregulation, eating healthier, and positively reframing the situation were the highest rated coping responses $(M=5.23$ to 6.25). Differences were found between BMI groups for intention to cope by suppressing appetite $(\mathrm{NW}=2.68 \pm 1.05, \mathrm{OWOB}=3.56 \pm 1.02 ; t=2.24, p<.05)$, supplement use (NW = 1.14 \pm .22 , OWOB $=1.85 \pm 1.28 ; t=2.30, p<.05)$, and a trend for camouflaging body $(\mathrm{NW}=2.70 \pm .51$, OWOB $=3.14 \pm .99, t=$ $1.57, p=.06)$. Feelings of shame were correlated with intention to camouflage body $(r=.46, p=.01)$, while authentic pride trended toward a negative correlation with suppressing appetite $(r=-.31)$, camouflaging $(r=-.35)$, and supplement use (-.34). In conclusion, an acute 3D body image scan session was shown to produce variation in self-conscious emotions (shame, guilt, and pride) and coping choices between NW and $O W O B$ participants. These individual differences should be taken into consideration to better accommodate healthy behavior change following 3D imaging use. 